\title{
Café da manhã: caracterização, consumo e importância para a saúde ${ }^{1}$
}

\author{
Breakfast: characterization, consumption \\ and importance for health
}

Suelen Caroline TRANCOSO ${ }^{2}$

Suzi Barletto CAVALLI²

Rossana Pacheco da Costa PROENÇA²

RE S U M O

O café da manhã é uma das três principais refeições do dia, mas, apesar da sua importância para a saúde, a diminuição do seu consumo é uma modificação importante no comportamento alimentar atual. Neste estudo, reflete-se sobre a caracterização dessa refeição e a implicação de seu consumo para a saúde; discutem-se, também, sua importância e as recomendações de consumo. Após revisão de artigos e respectivas contribuições científicas em bases de dados da área, pode-se afirmar que o café da manhã, objeto desta comunicação, é ainda bastante carente de pesquisas na literatura científica. Contudo, evidências científicas associam o consumo habitual de café da manhã a baixo risco de sobrepeso e obesidade, bem como melhoria na capacidade de aprendizagem. Estudos identificam que o perfil dos consumidores frequentes dessa refeição é de não fumantes que praticam atividade física, que controlam o peso e que não fazem uso frequente de álcool. Os autores pesquisados sugerem, assim, uma relação positiva entre o consumo de café da manhã e um estilo de vida saudável, justificando a recomendação de programas de incentivo ao seu consumo.

Termos de indexação: Alimentação básica. Comportamento alimentar. Estilo de vida.

A B S T R A C T

Breakfast is one of the main daily meals but despite its importance for health, skipping breakfast is becoming increasingly common and constitutes an important change from former to current food habits. This study discusses the characterization of this meal, its implication on health, its importance and consumption recommendations. Searching databases in the field for articles and their respective scientific contributions

\footnotetext{
1 Artigo elaborado a partir da dissertação de S.C. TRANCOSO, intitulada "Desenvolvimento de instrumento para avaliação da qualidade nutricional e sensorial de bufês de café da manhã”. Universidade Federal de Santa Catarina; 2008. Apoio: Coordenação de Aperfeiçoamento de Pessoal de Nível Superior e Conselho Nacional de Desenvolvimento Científico e Tecnológico (MCT/CNPq 02/2006, processo 478962/2006-1).

2 Universidade Federal de Santa Catarina, Programa de Pós-Graduação em Nutrição, Núcleo de Pesquisa de Nutrição em Produção de Refeições. Campus Universitário, Trindade, 88040-900, Florianópolis, SC, Brasil. Correspondência para/Correspondence to: R.P.C. PROENÇA. E-mail: <rossana@mboxl.ufsc.br>.
} 
860 | S.C. TRANCOSO et al.

evidences that there are very few studies on this meal. However, the existing studies show that regular breakfast consumption is associated with a lower risk of overweight and obesity and improved learning ability. Individuals who have breakfast regularly are usually physically active nonsmokers who monitor their weight and drink only sporadically. Thus, the reviewed articles suggest that there is a positive association between having breakfast regularly and healthy lifestyle, justifying the recommendation of programs that stimulate its consumption.

Termos de indexação: Staple Food. Feeding behavior. Life style.

\section{N T R O D U Ç Ã O}

Reconhecendo a importância da alimentação para a saúde, o Guia alimentar para a população brasileira considera saudável a refeição preparada com alimentos variados, com tipos e quantidades adequadas. Sugere pelo menos três refeições por dia - café da manhã, almoço e jantar -, intercaladas por pequenos lanches.

Apesar de o café da manhã ser considerado uma das principais refeições do dia, observa-se uma relativa carência de informações na literatura científica sobre ele quando comparado às outras refeições. Assim, neste estudo, a partir das contribuições científicas encontradas na literatura, reflete-se sobre a caracterização dessa refeição e a implicação de seu consumo para a saúde, discutindo-se, ainda, sua importância e recomendações de consumo.

\section{MÉTODOS}

Para a estruturação deste estudo foi realizada uma revisão de artigos nas bases de dados ScienceDirect, Scopus, SciELO, MedLine/PubMed, bem como no Portal de teses e dissertações da Capes, em livros e dicionários, considerando o período de 1996 a 2009. As palavras-chave pesquisadas foram "café da manhã", "desjejum" e "comportamento alimentar", nas línguas portuguesa e inglesa. Na análise das publicações, agruparam-se as informações de modo a caracterizar e a apresentar as definições de café da manhã, a diminuição do seu consumo, o impacto desse consumo para a saúde, os alimentos consumidos e os programas de incentivo ao café da manhã. A pesquisa não se pautou pela preocupação numérica de garantir a representatividade proporcional dos achados para análise quantitativa. Assim, privilegiando o enfoque de apresentar estudos relacionados ao mesmo tema, artigos semelhantes foram descartados.

\section{Definições do café da manhã}

O café da manhã é uma das três principais refeições do dia ${ }^{1-3}$, definida como a primeira refeição consumida pela manhã4. Um sinônimo para a expressão "café da manhã" é a palavra desjejum, que vem do latim e significa o rompimento do jejum involuntário mantido durante o sono ${ }^{4}$.

Quanto ao seu conteúdo, a recomendação brasileira é que ele garanta em média 25\% do total energético consumido durante o dia ${ }^{5}$. Esse percentual aproxima-se do valor encontrado por Requejo et al. ${ }^{6}$ e Rivas et al. ${ }^{7}$, que, ao investigarem adolescentes da Espanha que consomem frequentemente essa refeição, encontraram um consumo médio de 20\% do total energético diário.

Siega-Riz et al. ${ }^{8}$ e Rampersaud ${ }^{9}$ contribuem $^{2}$ para a definição do café da manhã, ressaltando quatro aspectos: a percepção que o indivíduo tem dessa refeição, o tipo de alimento frequentemente consumido (por exemplo: café com leite, pão com manteiga), a realização da refeição em uma hora específica do dia (por exemplo, às 7h da manhã), e o fato de ser a primeira refeição consumida após o acordar.

Estudo de Garcia ${ }^{10}$, no Brasil, analisando os discursos dos funcionários administrativos da região central de São Paulo, constatou que, quando questionados sobre sua alimentação, o café da manhã foi citado com uma frequência menor do que o almoço e o jantar. Para muitos, o café da manhã consistia em consumir somente o café, 
representando para poucos uma refeição mais completa. A percepção dos indivíduos entrevistados quanto ao café da manhã demonstra uma relação de certo descaso para com essa refeição, o que justifica a sugestão dessa autora quando utiliza a expressão "grandes refeições" para referir-se apenas aos eventos de almoço e jantar ${ }^{11}$.

\section{Diminuição no consumo de café da manhã}

O estilo de vida da sociedade contemporânea tem modificado os hábitos alimentares da população. No vasto campo de estudos sobre alimentação contemporânea, há evidências da diminuição do consumo de café da manhã como uma modificação importante no comportamento alimentar atual7 ${ }^{7-9,12-20}$.

Com objetivo de demonstrar a relevância do tema e correspondentes estudos em diferentes populações e faixas etárias, o (Quadro 1) apre- senta alguns estudos que tratam dessa preocupação com a diminuição significativa e frequente do café da manhã.

Autores mencionam que o declínio no consumo de café da manhã está normalmente relacionado com mudanças no estilo de vida contemporâneo da população, tais como: aumento do número de indivíduos que moram sozinhos, falta de tempo para realizar as refeições e particularidades no consumo de pratos diferentes pelos membros da família $8,16,21$.

Uma relação importante entre o consumo de café da manhã e a idade é também evidenciada nessas pesquisas. O consumo dessa refeição parece aumentar com a idade quando se trata de adul-

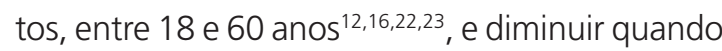
se estuda o consumo dessa refeição em crianças e adolescentes, entre 4 e 18 anos $^{2,8,9}$, constatando-se ainda que, na idade adulta, em geral, o consumo de café da manhã é predominantemente maior que em outras fases da vida.

Quadro 1. Estudos sobre a frequência do consumo de café da manhã entre crianças/adolescentes e adultos: localização, população pesquisada e percentagem do não consumo desta refeição.

\begin{tabular}{|c|c|c|c|}
\hline Autores & País & População pesquisada & $\begin{array}{c}\text { Não-consumo de café } \\
\text { da manhã }(\%)\end{array}$ \\
\hline \multicolumn{4}{|c|}{ Crianças e adolescentes } \\
\hline \multirow[t]{2}{*}{ Siega-Riz et al. ${ }^{8}$} & EUA & 7513 Adolescentes e crianças (1965) & $7 \%(1965)$ \\
\hline & & 4289 Adolescentes e crianças (1991) & $13 \%(1991)$ \\
\hline Gambardella et al. ${ }^{13}$ & Brasil & 153 Adolescentes & $55 \%$ \\
\hline Vieira et al. ${ }^{14}$ & Brasil & 185 Adolescentes & $37 \%$ \\
\hline \multirow[t]{3}{*}{ Nicklas et al. ${ }^{15}$} & EUA & 185 Crianças (1973-74) & $8 \%(1973-74)$ \\
\hline & & 158 Crianças (1978-79) & $30 \%(1978-79)$ \\
\hline & & 216 Crianças (1993-94) & $13 \%(1993-94)$ \\
\hline Rivas et al. ${ }^{7}$ & Espanha & 403 Adolescentes e crianças & $5 \%$ \\
\hline Sturion et al. ${ }^{17}$ & Brasil & 2678 Adolescentes e crianças & $19,5 \%$ \\
\hline \multirow[t]{2}{*}{ Unusan et al. ${ }^{18}$} & Turquia e & 460 Adolescentes (Turquia) & $4 \%$ \\
\hline & Alemanha & 422 Adolescentes (Alemanha) & $17,4 \%$ \\
\hline Wilson et al. ${ }^{19}$ & Nova Zelandia & 3275 Adolescentes e crianças & $16 \%$ \\
\hline \multicolumn{4}{|c|}{ Adultos } \\
\hline \multirow[t]{3}{*}{ Haines et al. ${ }^{12}$} & EUA & 6274 Adultos (1965) & $14,3 \%(1965)$ \\
\hline & & 18033 Adultos (1977-78) & $24,1 \%(1977-78)$ \\
\hline & & 10812 Adultos (1989-91) & $25,2 \%(1989-91)$ \\
\hline Song et al. ${ }^{16}$ & EUA & 4218 Adultos & $23 \%$ \\
\hline Alves \& Boog 20 & Brasil & 100 Adultos & $30 \%$ \\
\hline
\end{tabular}


A relação entre café da manhã e gênero foi também evidenciada em diferentes pesquisas: a omissão dessa refeição foi encontrada principalmente em adolescentes do sexo feminino. Entre as razões para esse achado, autores destacam a preocupação com a imagem corporal, o que comumente leva a pessoa a seguir dietas restritivas sem orientação, situação em que a prática de omitir refeições é muito comum ${ }^{9,14,24}$.

Outros estudos ressaltam que a omissão de refeições é um fenômeno comum entre adolescentes, para quem o café da manhã é a refeição mais negligenciada, o que se justifica pela irregularidade alimentar comum nessa fase da vida, seja pela atitude de submissão a dietas restritivas sem acompanhamento, seja pela referida falta de tempo para a realização de refeições ${ }^{2,13,17}$.

Entre as crianças, as razões normalmente citadas para a não realização do café da manhã são: falta de tempo, falta de fome nesse momento do dia ou desejo de fazer dieta para perder peso². Acredita-se que a falta de incentivo para a criação de um hábito alimentar que inclua o consumo de café da manhã também influencia a omissão dessa refeição pelas crianças.

Rampersaud et al. ${ }^{2}$, em estudo de revisão, também constataram uma alta prevalência de crianças e adolescentes que omitem o consumo de café da manhã, tanto nos Estados Unidos como na Europa (variando de 10\% a 30\%). Os autores revisaram 47 estudos, o que lhes possibilitou traçar um perfil dos não consumidores dessa refeição: adolescentes meninas, crianças de baixo nível socioeconômico, crianças e adolescentes mais velhos, bem como jovens negros e hispânicos. A omissão dessa refeição entre os adultos foi associada a pessoas fumantes, pessoas com baixa frequência de atividade física, pessoas que, preocupadas com o peso corporal, fazem dietas sem acompanhamento. Alexander et al. ${ }^{25}$ identificaram, ainda, associação positiva entre a omissão desta refeição e o aumento da adiposidade visceral.

No estudo de Keski-Rahkonen et al. ${ }^{24}$, diversos fatores foram significativamente associados ao perfil de adolescentes e adultos que não consomem café da manhã: fumar, não praticar exercícios físicos com frequência, apresentar baixo nível educacional, fazer uso frequente de álcool e ter alto Índice de Massa Corporal (IMC). Esses autores observaram, também, que as mulheres adolescentes e os homens adultos omitem mais frequentemente essa refeição.

Assim, alguns estudos mostram que o perfil dos consumidores habituais do café da manhã pode ser definido como sendo composto pela maioria de não fumantes, de pessoas que praticam atividade física, que não fazem uso frequente de álcool e que controlam o peso. Os estudos analisados parecem indicar uma relação positiva entre o consumo de café da manhã e um estilo de vida saudável|2,16,24,25.

\section{Impacto do consumo do café da manhã para a saúde}

Os achados dos estudos analisados foram agrupados pela relação entre o consumo do café da manhã e diferentes situações; refletindo-se sobre o impacto para a saúde, analisaram-se as vantagens do consumo e as desvantagens do não consumo dessa refeição para as pessoas que, omitindo-a, transformam em primeira refeição do dia um lanche no decorrer da manhã ou o almoço.

O Quadro 2 ilustra essa análise sobre os estudos que avaliaram as vantagens do consumo frequente e as desvantagens do não consumo de café da manhã. Para melhor visualização, os estudos são apresentados em ordem cronológica.

Evidências científicas relacionam o consumo frequente de café da manhã com baixo risco de sobrepeso e obesidade $2,3,15,16,23,25-31$.

O consumo frequente e adequado do café da manhã pode melhorar o poder de saciedade do comensal e, assim, reduzir a quantidade calórica total ingerida durante o dia; em especial, pode limitar o consumo de lanches calóricos por crianças e adolescentes ao longo da jornada ${ }^{31}$. No Brasil, Gauche et al. ${ }^{33}$, ao analisarem os ritmos circadianos de consumo de lanches e refeições 
Quadro 2. Estudos que avaliaram as vantagens do consumo frequente e as desvantagens do não consumo de café da manhã.

\begin{tabular}{|c|c|c|c|}
\hline \multicolumn{2}{|c|}{$\begin{array}{l}\text { Consumo frequente de café da } \\
\text { manhã apresentou relação com... }\end{array}$} & \multicolumn{2}{|c|}{$\begin{array}{l}\text { O não consumo de café da } \\
\text { manhã apresentou relação com... }\end{array}$} \\
\hline $\begin{array}{l}\text {...diminuição do risco de } \\
\text { sobrepeso e de obesidade }\end{array}$ & $\begin{array}{l}\text { Nicklas et al. }{ }^{26} \\
\text { Berkey et al.. } \\
\text { Sungsoo et al. }{ }^{28} \\
\text { Song et al. }{ }^{16} \\
\text { Affenito et al. }{ }^{23} \\
\text { Rampersaud et al. }{ }^{2} \\
\text { Niemeier et al.. } \\
\text { Affenito } \\
\text { Benton \& Jarvis }{ }^{30} \\
\text { Utter et al. }{ }^{31}\end{array}$ & $\begin{array}{l}\text {...aumento do consumo de } \\
\text { lanches calóricos (aumentar } \\
\text { o consumo energético total } \\
\text { de carboidratos e gorduras) }\end{array}$ & $\begin{array}{l}\text { Nicklas et al. }{ }^{26} \\
\text { Berkey et al. }{ }^{27} \\
\text { Sungsoo et al. }{ }^{28} \\
\text { Song et al. }{ }^{16} \\
\text { Affenito et al..23 } \\
\text { Rampersaud et al. } \\
\text { Niemeier et al. }{ }^{29} \\
\text { Affenito } \\
\text { Benton \& Jarvis }{ }^{30} \\
\text { Utter et al. }{ }^{31}\end{array}$ \\
\hline
\end{tabular}

em adultos, chegam à mesma constatação quando, ao apontarem a diminuição da frequência das três principais refeições - café da manhã, almoço e jantar -, sugerem que essas refeições podem estar sendo substituídas por pequenos lanches durante o dia. A substituição do café da manhã por lanches durante o dia, no entanto, não é vista como uma prática saudável. Alguns estudos mostram que essa substituição - principalmente em crianças e adolescentes comparados com indivíduos que ingeriam habitualmente o café da manhã - teve como consequência o aumento do consumo energético total de carboidratos - especialmente os açúcares simples - e de gorduras $^{7,15,17,30 \text {. }}$.

O consumo adequado do café da manhã parece também auxiliar no controle de peso; comparada aos lanches, a refeição matinal proporciona uma maior ingestão de vitaminas e minerais e menor ingestão de gorduras e colesterol ${ }^{31}$.

A ausência dessa refeição pode, por sua vez, inviabilizar a elevação da glicemia, necessária às atividades matinais, e favorecer uma possível deficiência de cálcio, uma vez que nessa refeição geralmente se concentra o maior consumo diário de leites e derivados, que são fontes desse mineral'13.
Outra relação importante pode ser feita entre o consumo de café da manhã e a melhoria no rendimento escolar de estudantes. Estudos apontaram efeitos positivos no desempenho cognitivo acadêmico 23,16,27,30,32, na atenção e na memória para atividades escolares ${ }^{2,29,31}$, e na frequência escolar de crianças e adolescentes².

Ao analisar o comportamento escolar de crianças, Benton \& Jarvi s $^{30}$ constataram que os que comem frequentemente café da manhã despendem mais tempo nos estudos do que os não consumidores dessa refeição; consequentemente, esses alunos apresentaram melhor rendimento escolar. Justifica-se, assim, a importância da existência de programas de café da manhã em escolas e a real necessidade de se incentivar o consumo dessa refeição em crianças e adolescentes 3,18,27,30.

Unusan et al. ${ }^{18}$, ao investigarem a percepção de estudantes quanto ao café da manhã, verificaram que, quando questionados sobre o sentimento que o consumo dessa refeição causava, a maioria relatou alegria e um sentimento de disposição após o seu consumo. Porém, os sentimentos de fraqueza e cansaço - relatados com frequência pelos não consumidores de café da manhã - não foram citados pelos consumidores dessa refeição. 


\section{Alimentos do café da manhã}

Vários autores destacam que a ingestão adequada do café da manhã pode representar a um indivíduo a oportunidade diária de consumir uma refeição com alimentos ricos em nutrientes e, assim, contribuir para uma dieta saudável $^{2,3,16,18,23,25-32}$.

Nas áreas urbanizadas do mundo ocidental, o café da manhã é realizado, normalmente, entre as 5 horas e as 10 horas da manhã ${ }^{12}$. No que se refere à quantidade calórica, Song et al. ${ }^{16}$ evidenciaram, em seu estudo, um consumo médio de $416 \mathrm{kcal}$ entre os consumidores habituais de café da manhã, o que representou um consumo de $18,6 \%$ do total energético diário. Quanto aos nutrientes dessa refeição, Nicklas et al. ${ }^{26}$ encontraram, entre os consumidores frequentes de café da manhã, um consumo médio de $13,0 \%$ de proteínas, $55,0 \%$ de carboidratos $(14,0 \%$ de açúcar simples) e $34,0 \%$ de gordura $(12,0 \%$ de gordura saturada).

No que se refere à quantidade calórica, o Guia Alimentar para a População Brasileira' sugere um consumo médio diário de 2 mil kcal totais. Philippi ${ }^{5}$ recomenda dividir esse Valor Energético Total (VET) diário em seis refeições, cabendo ao café da manhã um consumo de $25 \%$ do VET, ou seja, um consumo médio de $500 \mathrm{kcal}$.

No Brasil, a composição dessa refeição pode ser simplificada pelo consumo de café com leite e pão com manteiga ou margarina ${ }^{11}$; ou, no caso de uma composição mais completa: leite, café, pães, frios, biscoitos, frutas e sucos de frutas, manteiga/margarina ${ }^{34}$. Essa composição é semeIhante à encontrada no estudo de Mattos \& Martins ${ }^{35}$, que em uma amostra de 559 indivíduos com mais de 20 anos do município de Cotia, São Paulo, Brasil, identificaram como alimentos mais consumidos no café da manhã: café (87,5\%), pão francês $(70,8 \%)$, leite (51,3\%) e margarina (50,8\%). O consumo de frutas foi referido por apenas $11,3 \%$ da população, sendo elas: Iaranja (7,2\%) e banana nanica $(4,1 \%)$.
Gambardella et al..$^{13}$ sugerem uma composição padrão para o café da manhã de adolescentes brasileiros, constituída de alimentos fonte de cálcio e de energia. A fonte de cálcio sugerida é o leite e seus derivados; a fonte energética, por sua vez, é constituída de pães e biscoitos, com acompanhamentos como geleias, mel, margarinas, manteiga, maionese, queijos e frios.

Alves \& Boog ${ }^{20}$, adaptando as opções de Gambardella et al. ${ }^{13}$, sugerem três configurações de café da manhã: padrão, completo e incompleto. O café da manhã padrão manteve-se como a definição original. A configuração café da manhã completo foi definida como uma refeição composta por alimentos fonte de cálcio, de energia e alimentos reguladores. Já o café da manhã incompleto seria uma refeição com quaisquer outros alimentos que não contemplem as combinações apresentadas nas opções padrão ou completo.

Sungsoo et al. ${ }^{28}$ separam a configuração do café da manhã em nove grupos de alimentos frequentemente consumidos nos Estados Unidos da América por diferentes etnias, que são: 1) gorduras e doces, 2) produtos lácteos, 3) carnes e ovos, 4) frutas e vegetais, 5) cereais matinais, 6) cereais cozidos, 7) pães, 8) bolos, tortas, panquecas, waffles e 9) bebidas. Dentre esses, os mais consumidos foram: os grupos de cereais matinais, pães, bolos, tortas, panquecas, waffles, carnes e ovos.

Estudos mencionam que a qualidade nutricional do café da manhã nos Estados Unidos da América melhorou nos últimos anos, provavelmente devido ao aumento da oferta e do consumo de alimentos considerados mais saudáveis, como, por exemplo, cereais matinais e iogurtes, paralelamente à diminuição do consumo de gordura, encontrada sobretudo no bacon e nos $\operatorname{ovos}^{8,12,16,22,36}$. Pesquisas demonstram que nesse país, no período de 1965 a 1996, no café da manhã de adultos, adolescentes e crianças, houve um aumento no consumo de leite desnatado, pães integrais, cereais ricos em fibras, e frutas cítricas. Como contraponto, houve diminuição no 
consumo de leite integral, bacon, ovos, pão branco, pão branco rico em gordura, manteiga e margarina ${ }^{8,12}$.

Ainda nos Estados Unidos da América, o consumo de cereal matinal tem sido alvo de diversos estudos, uma vez que a ingestão de cereais está sendo relacionada com benefícios para a saúde: redução no consumo total de gorduras, diminuição nos níveis de colesterol sérico e índice glicêmico; aumento no consumo de fibras, de magnésio e vitamina $\mathrm{E}^{8,12,36}$. Carson et al. ${ }^{22}$ corroboram essa afirmação ao evidenciarem que os indivíduos que consomem regularmente (mais de três dias por semana) cereal matinal no café da manhã têm as proporções da gordura total, da gordura saturada e do colesterol mais baixas que os não consumidores. Apresentam, ainda, as proporções de fibra, cálcio, ferro, zinco, e das vitaminas $A, B, C$ e E mais elevadas. No estudo de Song et al. ${ }^{36}$, o consumo desse cereal foi associado ao hábito de realizar a refeição matinal e à ingestão de leite, o que colaborou para que a amostra dos consumidores do cereal tivesse ingestão adequada de cálcio.

Os estudos acima citados referem-se aos cereais matinais descritos em inglês como "Ready-To-Eat Cereal - RTEC" 8,12,22,36, não explicitando, na maioria das vezes, a que tipo de cereal matinal específico se refere. Assim, essa afirmação de benefícios para a saúde deve ser encarada com cuidado, considerando-se que, no Brasil, encontram-se, minimamente, dois tipos de cereais matinais cujo consumo teria potencialmente consequências distintas para a saúde. Um grupo, por exemplo, seria formado pelos cereais matinais elaborados com farinhas e açúcar refinados, adicionados de vitaminas e minerais, sendo, portanto, fontes de carboidratos simples e micronutrientes artificiais. Outro grupo seria o dos cereais matinais elaborados com farinhas integrais, oleaginosas e açúcar mascavo, sendo fontes de carboidratos complexos, fibras, vitaminas e minerais.

Poulain ${ }^{37}$, ao analisar as características dos hábitos alimentares contemporâneos na França, descreve que o café da manhã mais tradicional consiste basicamente no consumo de uma bebida quente e um pão com manteiga ou croissant, sugerindo, assim, uma refeição mais simplificada. Michaud et al. ${ }^{38}$ e Lambert et al..$^{21}$ corroboram essa descrição ao sugerir que, além do café da manhã, o almoço e o jantar francês também sofreram modificações no decorrer dos anos, apresentando-se hoje como refeições mais simplificadas.

Na Espanha, o café da manhã considerado adequado deve ser composto por leite, cereais, torradas com azeite, frutas in natura e/ou suco de frutas $^{38,39}$.

O Quadro 3 aponta os estudos encontrados e os correspondentes alimentos investigados que integram o café da manhã em alguns países, apresentando um panorama das diferenças estruturais dessa refeição. Pode-se observar a ingestão quase generalizada de algum tipo de leite, de pão e de cereais, demonstrando certa homogeneidade na composição dessa refeição em regiões urbanas de países do ocidente.

Rampersaud et al. ${ }^{2}$ sugerem uma lista de recomendações sobre o consumo de café da manhã para crianças e adolescentes, destacando-se a necessidade de consumir diariamente um café da manhã saudável com variedade entre os grupos alimentares (por exemplo, grãos integrais, frutas e produtos lácteos), priorizando o consumo de alimentos nutritivos, ricos em fibras, pobres em açúcar e alimentos reguladores. O estudo ainda sugere que o incentivo dessa refeição para crianças e adolescentes deve acontecer sempre, tanto por parte dos pais como por parte das escolas e programas de incentivo ao consumo de café da manhã.

Nesse enfoque, Trancoso ${ }^{40}$ desenvolveu um instrumento de avaliação da qualidade nutricional e sensorial de bufês de café da manhã (AQCM). Esse instrumento pode ser utilizado, por exemplo, como forma de avaliar um café da manhã adequado, pois sugere um padrão mínimo para garantir o consumo dessa refeição com qualidade nutricional e sensorial, além de fornecer 
866 | S.C. TRANCOSO et al.

Quadro 3. Estudos sobre o café da manhã: localização, população estudada e alimentos investigados em diferentes países.

\begin{tabular}{|c|c|c|c|c|c|c|c|c|c|c|c|c|}
\hline & 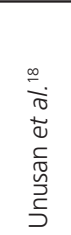 & 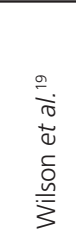 & 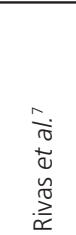 & 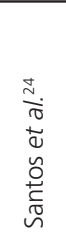 & 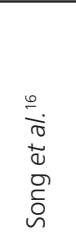 & 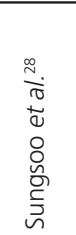 & $\begin{array}{l}\stackrel{0}{N} \\
\frac{N}{\pi} \\
\frac{N}{L} \\
\frac{1}{\alpha}\end{array}$ & 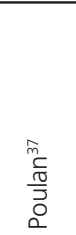 & 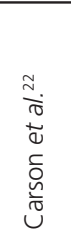 & 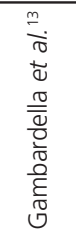 & 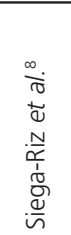 & 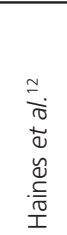 \\
\hline & 2006 & 2006 & 2005 & 2005 & 2005 & 2003 & 2002 & 2002 & 1999 & 1999 & 1998 & 1996 \\
\hline \multicolumn{13}{|l|}{ Localização } \\
\hline Estados Unidos & & & & & $x$ & $x$ & & & $x$ & & $x$ & $x$ \\
\hline Nova Zelândia & & $x$ & & & & & & & & & & \\
\hline Espanha & & & $x$ & & & & $x$ & & & & & \\
\hline França & & & & & & & & $x$ & & & & \\
\hline Turquia e Alemanha & $x$ & & & & & & & & & & & \\
\hline Brasil & & & & $x$ & & & & & & $x$ & & \\
\hline \multicolumn{13}{|l|}{ População estudada } \\
\hline Adolescentes ou crianças & $x$ & $x$ & $x$ & $x$ & & & & & & $x$ & & \\
\hline Adultos & & & & & $x$ & $x$ & $x$ & $x$ & $x$ & & $x$ & $x$ \\
\hline \multicolumn{13}{|l|}{ Alimentos investigados no café da manhã } \\
\hline Leite (integral, semi-desnatado e desnatado) & $x$ & $x$ & $x$ & $x$ & & $x$ & $x$ & & & $x$ & $x$ & $x$ \\
\hline $\begin{array}{l}\text { Pães (branco, integral, } \\
\text { com pouca gordura) }\end{array}$ & $x$ & $x$ & & $x$ & & $x$ & $x$ & $x$ & & $x$ & $x$ & $x$ \\
\hline $\begin{array}{l}\text { Cereais matinais (ricos ou não em fibras, RTE "read } \\
\text { to eat") }\end{array}$ & $x$ & $x$ & $x$ & & $x$ & $x$ & $x$ & & $x$ & & $x$ & $x$ \\
\hline Frutas e hortaliças (sucos ou in natura) & $x$ & $x$ & $x$ & $x$ & & $x$ & $x$ & & & $x$ & $x$ & $x$ \\
\hline Manteiga e margarina, mel e geleias. & $x$ & & & & & & & $x$ & & $x$ & $x$ & $x$ \\
\hline Carnes & & & & $x$ & & $x$ & & & & & $x$ & $x$ \\
\hline Frios (queijo, presunto e bacon) & $x$ & & & $x$ & & $x$ & & & & $x$ & & \\
\hline Ovos & $x$ & $x$ & & $x$ & & $x$ & & & & & $x$ & $x$ \\
\hline Biscoitos, tortas, bolos, panquecas e waffles & $x$ & & $x$ & $x$ & & $x$ & & & & $x$ & & \\
\hline Achocolatados & & & $x$ & & & & & & & $x$ & & \\
\hline
\end{tabular}

alternativas para melhorar a qualidade dessa refeição por meio de um plano de ação estruturado. $\mathrm{O}$ instrumento de avaliação desenvolvido respeita a oferta de alimentos saudáveis, regionais e da estação, as opções de melhorias, a forma de apresentação dos alimentos, o fornecimento de informações completas, ditas importantes para a saúde, dentre outros itens.

\section{Programas de incentivo ao café da manhã}

Constatados os benefícios do consumo adequado do café da manhã para a saúde, surgiram alguns programas que visam a educar e a incentivar crianças e adolescentes para o consumo dessa refeição de forma mais saudável, buscando formar bons hábitos para a vida adulta. Estudos relatam que tais programas podem ter a capacidade de diminuir a disparidade do consumo nutricional de alimentos e adequar a ingestão alimentar no café da manhã, além de contribuir para a diminuição de sobrepeso e obesidade e para a melhoria do rendimento escolar em crianças e adolescentes $2,3,9,15-19,23,27,30-32,41,42$.

Para que esse incentivo fosse eficiente, alguns programas buscaram educar crianças na fase escolar, como é o exemplo do programa School Breakfast Program (SBP) do Departamento de Agricultura dos Estados Unidos da América ${ }^{41}$. No México, um programa de café da manhã para escolares - Programa de Desayunos Escolares (PDE) -, além de incentivar a ingestão dessa 
refeição matinal, preocupou-se também em adequar nutricionalmente os alimentos nela oferecidos às crianças, incluindo alimentos fortificados, tais como: leite fortificado com vitamina A, cereais, bolachas e pães com ferro, sucos de frutas com vitamina $C$, dentre outros ${ }^{42}$.

No Brasil, o Programa Nacional de Alimentação Escolar (PNAE) oferece a chamada merenda escolar, que visa melhorar as condições nutricionais e a capacidade de aprendizagem de crianças que frequentem as escolas desde a pré-escola até o ensino fundamental da rede pública ou filantrópica. Destaca-se que o PNAE não se refere especificamente ao café da manhã, e sim ao fornecimento de lanches e almoço, embora seja o café da manhã a refeição mais comumente oferecida ${ }^{17,43}$.

\section{O N CLUS Ã O}

O Guia Alimentar para a População Brasileira ${ }^{1}$ sugere que as três principais refeições diárias - café da manhã, almoço e jantar - devam aproximar-se do atendimento das porções diárias recomendadas. Dessa forma, considerando a importância e as vantagens do seu consumo adequado, o café da manhã poderia ser responsável pelo consumo de algumas ou de todas as porções diárias de alguns grupos alimentares, como o dos cereais, o das frutas e sucos de frutas, e o do leite e derivados. Esse fato poderia contribuir para uma adequação nutricional no consumo de alimentos por parte da população, auxiliar na diminuição de sobrepeso e de obesidade e, ainda, melhorar o rendimento escolar, principalmente, em crianças e adolescentes.

Com base na revisão realizada e diante dos benefícios do consumo adequado do café da manhã, considera-se importante que os profissionais e os programas de saúde ligados à alimentação incrementem o incentivo para a realização dessa refeição, objetivando a criação de um hábito alimentar que a inclua. Atingido esse objetivo, outras contribuições à saúde poderão ser também alcançadas, uma vez que os estudos correlacionam o perfil dos consumidores habituais de café da manhã com um estilo de vida saudável.

Sabe-se que existem iniciativas brasileiras de incentivo ao consumo de café da manhã realizadas tanto por prefeituras municipais em restaurantes populares quanto em empresas cadastradas ou não ao Programa de Alimentação do Trabalhador (PAT). Contudo, como não foram encontrados estudos científicos analisando tais iniciativas, elas não puderam ser discutidas no contexto deste artigo.

Apesar de os estudos sugerirem o incentivo do consumo frequente e adequado do café da manhã como forma de garantir um estilo de vida saudável, observou-se a necessidade de reflexão e definição do que seria esse consumo adequado para a população de cada país, considerando modelos alimentares regionais. Outra questão seria discutir os significados dessa refeição em diferentes países, posto que, nos artigos analisados, nem mesmo a definição da refeição é comumente discutida, citando-a apenas como uma palavra de senso comum.

Por fim, é também importante a discussão sobre instrumentos de avaliação para essa refeição, imprescindíveis tanto ao meio científico como à vida cotidiana, considerando-se as especificidades do café da manhã e a necessidade cada vez maior da sua realização fora de casa.

\section{COLABORADORES}

S.C. TRANCOSO participou da elaboração do projeto de pesquisa, coleta e análise dos dados, discussão dos resultados e elaboração do artigo. R.P.C. PROENÇA e S.B. CAVALLI participaram da elaboração do projeto de pesquisa, análise dos dados, discussão dos resultados e elaboração do artigo.

\section{REFERÊ NCIAS}

1. Brasil. Ministério da Saúde. Guia alimentar para a população brasileira. Brasília: Ministério da Saúde; 2006.

2. Rampersaud GC, Pereira MA, Girard BL, Adams J, Metzl J. Breakfast habits, nutritional status, body 
weight, and academic performance in children and adolescents. J Am Diet Assoc. 2005; 105(5):743-60.

3. Affenito SG. Breakfast: a missed opportunity. J Am Diet Assoc. 2007; 107(4):565-9.

4. Houaiss A, Villar MS. Dicionário da língua brasileira. $2^{a}$ ed. Rio de Janeiro: Objetiva; 2004.

5. Philippi ST. Pirâmide dos alimentos: fundamentos básicos da nutrição. Barueri: Manole; 2008.

6. Requejo AM, Ortega RM, Lopez Sobaler AM, Quintas ME, Andrés P, Redondo MR, et al. Valeur nutritionnelle du petit déjeuner et composition de I'alimentation quotidienne chez des écoliers de Madrid. Cah Nutr Diét.1998; 33(1):41-7.

7. Rivas $P R$, Figuero CR, Lanza, TA, lamuño DG, Fuentes MG, Grupo Avena. Desayuno y almuerzo de los adolescentes escolarizados de Santander. Nutr Hosp. 2005; 20(3):217-22.

8. Siega-Riz AM, Popkin BM, Carson T. Trends in breakfast consumption for children in the United States from 1965 to 1991. Am J Clin Nutr. 1998; 67(Suppl):748S-56S.

9. Rampersaud GC. Benefits of breakfast for children and adolescents: update and recommendations for practitioners. Am J Lifestyle Medicine. 2009; 3(2): 86-103.

10. Garcia RWD. Práticas e comportamento alimentar no meio urbano: um estudo no centro urbano da cidade de São Paulo. Cad Saúde Pública. 1997; 13(3): 455-67.

11. Garcia RWD. Comida, a dieta, o gosto: mudanças na cultura alimentar urbana [tese]. São Paulo: Universidade de São Paulo; 1999.

12. Haines PS, Guilkey DK, Popkin BM. Trends in breakfast consumption if US adults between 1965 and 1991. J Am Diet Assoc. 1996; 96(5):464-70.

13. Gambardella AND, Frutuoso MPF, Franchi C. Prática alimentar de adolescentes. Rev Nutr. 1999; 12(1): 55-63. doi: 10.1590/S1415-52731999000100005.

14. Vieira VCR, Priore SE, Ribeiro SMR, Franceschini SCC, Almeida LP. Perfil socioeconômico, nutricional e de saúde de adolescentes recém-ingressos em uma universidade pública brasileira. Rev Nutr. 2002; 15(3):273-82. doi: 10.1590/S1415-5273200200 2000300003.

15. Nicklas TA, Morales M, Linares A, Yang S, Baranowski T, De Moor C, et al. Children's meal patterns have changed over a 21-year period: the Bogalusa heart study. J Am Diet Assoc. 2004; 104(5):753-61.

16. Song W, Chun O, Obayashi S, Cho S. Chung, C. Is consumption of breakfast associated with body mass index in US adults? J Am Diet Assoc. 2005; 105(9):1373-82.
17. Sturion GL, Silva MV, Ometto AMH, Furtuoso MCO, Pipitone MAP. Fatores condicionantes da adesão dos alunos ao Programa de Alimentação Escolar no Brasil. Rev Nutr. 2005; 18(2):167-81. doi: 10.15 90/S1415-52732005000200001.

18. Unusan N, Sanlier N, Danisik H. Comparison of attitudes towards breakfast by Turkish fourth graders living in Turkey and Germany. Appetite. 2006; 46(3):248-53.

19. Wilson NC, Parnell WR, Wohlers M, Shirley PM. Eating breakfast and its impact on children's daily diet. Nutr Diet. 2006; 63(1):15-9.

20. Alves HJ, Boog MCF. Comportamento alimentar em moradia estudantil: um espaço para promoção da saúde. Rev Saúde Pública. 2007; 41(2):197-204.

21. Lambert JL, Batalha MO, Sproesser RL, Silva AL, Lucchese T. As principais evoluções dos comportamentos alimentares: o caso da França. Rev Nutr. 2005;18(5):577-91. doi: 10.1590/S1415-5273200 5000500001.

22. Carson TA, Siega-Riz AM, Popkin BM. The importance of breakfast meal type to daily nutrient intake: Differences by age and ethnicity. Cereal Foods World. 1999; 44(6):414-22.

23. Affenito SG, Thompson DR, Barton BA, Franko DL, Daniels SR, Obarzanek E, et al. Breakfast consumption by African-American and white adolescent girls correlates positively with calcium and fiber intake and negatively with body mass index. J Am Diet Assoc. 2005; 105(6):938-45.

24. Keski-Rahkonen A, Kaprio J, Rissanen A, Virkkunen $M$, Rose RJ. Breakfast skipping and healthcompromising behaviors in adolescents and adults. Eur J Clin Nutr. 2003; 57(7):842-53.

25. Alexander KE, Ventura EE, Spruijt-Metz D, Weigensberg MJ, Goran MI, Davis JN. Association of breakfast skipping with visceral fat and insulin indices in overweight Latino youth. Obesity. 2009; 17(8):1528-33.

26. Nicklas TA, Myers L, Reger C, Beech B, Berenson GS. Impact of breakfast consumption on nutritional adequacy of the diets of young adults in Bogalusa, Luisiana: Ethic and gender contrasts. J Am Diet Assoc. 1998; 98(12):1432-8.

27. Berkey CS, Rockett HRH, Gillman MW, Field $A E$, Colditz GA. Longitudinal study of skipping breakfast and weight change in adolescents. Int J Obes. 2003; 27(10):1258-66.

28. Sungsoo C, Dietrich M, Brown CJP, Clarck CA, Block $G$. The effect of breakfast type on total daily energy intake and body mass index: results from the Third National Health and Nutrition Examination Survey (NHANES III). J Am Diet Assoc. 2003; 22(4):296-302.

29. Niemeier H, Raynor H, Lloyd-Richardson E, Rogers $M$, Wing R. Fast food consumption and breakfast 
skipping: predictors of weight gain from adolescence to adulthood in a nationally representative sample. J Adolesc Health. 2006; 39(6):842-9.

30. Benton $D$, Jarvis $M$. The role of breakfast and a midmorning snack on the ability of children to concentrate at school. Physiol Behav. 2007; 90(2-3): 382-5.

31. Utter J, Scragg R, Mhurchu C, Schaaf D. At-home breakfast consumption among New Zealand children: Associations with body mass index and related nutrition behaviors. J Am Diet Assoc. 2007; 107(4):570-6.

32. Moore GF, Tapper K, Murphy S, Lynch R, Raisanen $\mathrm{L}$, Pimm C, et al. Associations between deprivation, attitudes towards eating breakfast and breakfast eating behaviours in 9-to-11-year-olds. Public Health Nutr. 2007; 10(6):582-9.

33. Gauche H, Calvo MCM, Assis MAA. Ritmos circadianos de consumo alimentar nos lanches e refeições de adultos: aplicação do semanário alimentar. Nutr. 2006; 19(2):177-85. doi: 10.1590/\$14 15-52732006000200005.

34. Santos JS, Costa COM, Sobrinho CLN, Silva MCM, Souza KEP, Melo BO. Perfil antropométrico e consumo alimentar de adolescentes de Teixeira de Freitas - Bahia. Rev Nutr. 2005; 18(5):623-32. doi: 10.15 90/S1415-52732006000500005.

35. Mattos LL, Martins IS. Consumo de fibras alimentares em população adulta. Rev Saude Pública. 2000; 34(1):50-5.

36. Song W, Chun O, Kerver J, Cho S, Chung C, Chung S. Ready-to-eat breakfast cereal consumption enhances milk and calcium intake in the US population. J Am Diet Assoc. 2006; 106(11):1783-9.

37. Poulain JP. The contemporary diet in France: "destructuration" or from commensalism to "vagabond feeding". Appetite. 2002; 39:43-55.

38. Michaud C, Baudier F, Guilbert P, Carel D, Le Bihan G, Gautier A, et al. Les repas des français: résultats du baromètre santé nutrition 2002. Cah Nutr Diet. 2004; 39(3):203-9.

39. Arnáiz MG. Somos lo que comemos. Barcelona: Ariel; 2002.

40. Trancoso SC. Desenvolvimento de instrumento para avaliação da qualidade nutricional e sensorial de bufês de café da manhã em hotéis de negócios [dissertação]. Florianópolis: Universidade Federal de Santa Catarina; 2008.

41. Kennedy E, Davis C. US Department of Agriculture School Breakfast Program. Am J Clin Nutr.1998; 67(Suppl):798S-803S.

42. Lopez ER, Haro MI, Valencia ME, Ponce JA, Artalejo E. Impacto de un programa de desayunos escolares en la prevalecía de obesidad y factores de riesgo cardiovascular en niños sonorenses. Salud Publica Mex. 2005; 47(2):126-33.

43. Muniz VM, Carvalho AT. O programa de alimentação escolar em município do estado da Paraíba: um estudo sob o olhar dos beneficiários do programa. Rev Nutr. 2007; 20(3):285-96. doi: 10.1590/ S1415-52732007000300007.

Recebido em: 11/6/2008

Versão final reapresentada em: 4/5/2010 Aprovado em: 31/5/2010 
